\title{
Re-defining the stress cortisol response to surgery
}

\author{
Abbreviated Title: Stress Cortisol Response to Surgery \\ Bernard Khoo*1 ${ }^{1}$, Piers R Boshier*2, Alexander Freethy ${ }^{3}$, George Tharakan ${ }^{3}$, Samerah Saeed $^{2}$, Neil Hill ${ }^{3}$, Emma \\ L Williams ${ }^{4}$, Krishna Moorthy ${ }^{5}$, Neil Tolley ${ }^{5}$, Long R Jiao ${ }^{5}$, Duncan Spalding, Fausto Palazzo ${ }^{6}$, Karim \\ Meeran $^{3}$, Tricia $\operatorname{Tan}^{\dagger 3}$
}

${ }^{1}$ Endocrinology, Division of Medicine, University College London, Royal Free Hospital, London, UK

${ }^{2}$ Department of Surgery and Cancer, Imperial College London, St Mary's Hospital, London, UK

${ }^{3}$ Section of Investigative Medicine, Division of Diabetes, Endocrinology and Metabolism

Imperial College London, Hammersmith Campus, London, UK

${ }^{4}$ Department of Clinical Biochemistry, Imperial College Healthcare NHS Trust, Charing Cross Hospital, London, UK

${ }^{5}$ Department of Surgery, Imperial College Healthcare NHS Trust, Hammersmith Hospital, London, UK

${ }^{6}$ Department of Endocrine Surgery, Imperial College Healthcare NHS Trust, Hammersmith Hospital, London, UK

*These authors contributed equally to this work

\section{Word Count: 3796}

\section{Number of Figures and Tables: 6}

\section{Corresponding Author and person to whom reprint requests should be addressed}

\section{Prof Tricia Tan, MB ChB BSc PhD FRCP FRCPath}

Section of Investigative Medicine

Division of Diabetes, Endocrinology and Metabolism

Imperial College London

$6^{\text {th }}$ Floor, Commonwealth Building

Du Cane Road 
London W12 0HS

Tel: 02033138038

e-mail: t.tan@imperial.ac.uk

\section{ACKNOWLEDGEMENTS AND FUNDING}

The Section of Endocrinology and Investigative Medicine is funded by grants from the UK Medical Research Council (MRC), UK Biotechnology and Biological Sciences Research Council (BBSRC), UK National Institute for Health Research (NIHR), an Integrative Mammalian Biology (IMB) Capacity Building Award, an FP7-HEALTH-2009-241592 EuroCHIP grant and is supported by the NIHR Biomedical Research Centre Funding Scheme. The authors would also like to thank the anaesthetiologists and surgeons at Imperial Healthcare NHS Trust who permitted the research team to collect samples during the study.

Disclosure Summary: The authors have nothing to disclose 


\section{ABSTRACT}

\section{Background}

Cortisol levels rise with the physiological stress of surgery. Previous studies have used older, less-specific assays, have not differentiated by severity, or only studied procedures of a defined type. The aim of this study was to examine this phenomenon in surgeries of varying severity using a widely used cortisol immunoassay.

\section{Methods}

Euadrenal patients undergoing elective surgery were enrolled prospectively. Serum samples were taken at 8 am on surgical day, induction and $1 \mathrm{hr}, 2 \mathrm{hr}, 4 \mathrm{hr}$ and $8 \mathrm{hr}$ after. Subsequent samples were taken daily at 8 am until post-operative day 5 or hospital discharge. Total cortisol was measured using an Abbott Architect immunoassay, and cortisol binding globulin ( $\mathrm{CBG})$ using a radioimmunoassay. Surgical severity was classified by POSSUM operative severity score.

\section{Results}

93 patients underwent surgery: Major/Major+ $(n=37)$, Moderate $(n=33)$, and Minor $(n=23)$. Peak cortisol positively correlated to severity: Major/Major+ median 680 [range 375-1452], Moderate 581 [270-1009], and Minor 574 [272-1066] nmol/L (Kruskal-Wallis test, $P=0.0031$ ). CBG fell by 23\%; the magnitude of the drop positively correlated to severity.

\section{Conclusions}

The range in baseline and peak cortisol response to surgery is wide, and peak cortisol levels lower than previously appreciated. Improvements in surgery, anaesthetic techniques, and cortisol assays might explain our observed lower peak cortisols. The criteria for the dynamic testing of cortisol response may need to be reduced to take account of these factors. Our data also support a lower-dose, stratified approach to dosing of steroid replacement in hypoadrenal patients, to minimise the deleterious effects of over-replacement.

Key terms: Cortisol; Stress Response; Surgery; Cortisol Binding Globulin 


\section{INTRODUCTION}

Trauma and invasive surgery are associated with a stress response that varies in accordance with the nature and degree of tissue damage. This physiological response to allows the body to react in a coordinated manner to traumatic stimuli and acts as a mechanism to attenuate further tissue damage. Critical to this response is the release of cortisol, mediated by the Hypothalamo-Pituitary-Adrenal axis ${ }^{1}$.

The effects of cortisol in the setting of surgical stress are complex, but include: suppression of insulin and mobilisation of energy stores (gluconeogenesis, glycogenolysis); increased proteolysis; sodium and water retention leading to preservation of blood pressure; suppression of the immune-inflammatory response; and delayed wound healing through its effects on collagen synthesis. Measured serum cortisol levels reflect total cortisol, of which approximately $80 \%$ is bound to cortisol binding globulin (CBG) and $10 \%$ to albumin. Therefore, only a small fraction of serum cortisol remains unbound in a biologically active form ${ }^{2}$.

Much of our understanding of the perioperative adrenocortical response relates to research conducted half a century ago by Plumpton and Besser ${ }^{3}$. Whilst subsequent studies have measured cortisol in the perioperative setting as a marker of surgical stress ${ }^{4-10}$, these studies are subject to certain limitations. First, the older studies have used cortisol assays which are now known to be non-specific and which are generally positively biased in comparison to modern assays aligned to reference methods; second, the studies have usually looked at very specific types of surgery, e.g. cardiovascular surgery ${ }^{5}$, colorectal surgery ${ }^{6}$, arthroscopy ${ }^{7}$, or cholecystectomy ${ }^{10}$; third, these studies have generally studied types of surgery which are at the major end of the scale, and not systematically investigated the cortisol response to surgeries of different severity; fourth, these studies have not systematically investigated the CBG response to surgeries of different severity, which would be expected to modify the impact of the cortisol response. An appreciation of the expected endocrine stress response to surgeries of varying severity is important as it will further our understanding of the physiological response to controlled trauma, and inform the care of hypoadrenal patients during surgery. The aim of the current study was therefore to measure the adrenocortical response to surgeries of varied severity. 


\section{MATERIALS AND METHODS}

Local ethics committee approval (London Riverside REC: ref. 14/10/0104) was obtained for this prospective cohort study and all patients provided informed written consent prior to enrolment.

Patients between the age of 18 and 90 years undergoing elective surgical procedures at St Mary's and Hammersmith Hospitals (Imperial College Healthcare NHS Trust) were invited to take part in the current study. Patients were principally recruited from within the departments of endocrine surgery, upper gastrointestinal surgery (including hepatobiliary and pancreatic surgery) and cardiothoracic surgery. All patients underwent general anaesthesia. None underwent surgery with etomidate anaesthesia, which is known to inhibit cortisol biosynthesis.

Patients were excluded from the study if they were known to be hypoadrenal, to be pregnant (confirmed by a positive urine $\beta$-hCG test), or if they had consumed any drug within the preceding month which is known to affect the HPA axis or measurement of total serum cortisol, for example oral oestrogens, systemic or topical glucocorticoids.

Serum cortisol was measured at 8 am on the day of surgery, at anaesthetic induction and at $1 \mathrm{hr}, 2 \mathrm{hr}, 4 \mathrm{hr}$, and $8 \mathrm{hr}$ thereafter. Cortisol ( $8 \mathrm{am})$ was then measured daily until post-operative day five, or discharge, whichever was the earlier. Total serum cortisol was measured using an automated Abbott Architect immunoassay, an assay which has been shown to correlate well to reference GC-MS methods ${ }^{11}$. The precision was $\leq 10 \%$ total coefficient of variation (CV) for serum samples $\geq 83$ to $\leq 965 \mathrm{nmol} / \mathrm{L}$, the limit of detection was $\leq 22 \mathrm{nmol} / \mathrm{L}$. The cross-reactivity of cortisone in this assay is minimal $(2.7 \%$ at $1000 \mu \mathrm{g} / \mathrm{dl})$.

Serum cortisol binding globulin (CBG) was measured by immunoassay (Biosource Technologies, Inc. Europe S.A., Nivelles, Belgium). The intra-assay precision was $8.6 \% \mathrm{CV}$ at $23.1 \mathrm{mg} / \mathrm{L}$ and $3.9 \% \mathrm{CV}$ at $83.3 \mathrm{mg} / \mathrm{L}$. The inter-assay precision was $10.8 \% \mathrm{CV}$ at $24.7 \mathrm{mg} / \mathrm{L}, 4.8 \% \mathrm{CV}$ at $113.8 \mathrm{mg} / \mathrm{L}$. The free cortisol index (FCI) was calculated as the ratio of the total cortisol divided by the contemporaneous CBG level. 
Operative severity was classified as either Minor, Moderate, or Major/Major+, in accordance with the POSSUM operative severity scoring system ${ }^{12}$.

Statistical analysis was performed using the Prism 6.0h software package (GraphPad Inc., San Diego, USA). The D'Agostino-Pearson test was used to test for normality. Parametric data is presented as mean \pm standard deviation, and non-parametric data as median [95\% confidence interval of median, or range as indicated]. Statistical significance was assigned to two-tailed $P$-values of $<0.05$. In 13 cases, steroid injections (usually dexamethasone) were given either intraoperatively or at the end of the operation. The cortisol values for the time points after the injection were censored from analysis on the basis that the exogenous steroids would suppress the physiological cortisol response to stress, but the time points prior to the injection were included as these were informative as to the stress cortisol response. 


\section{RESULTS}

Ninety-three patients undergoing elective surgery were classified by surgical severity: Major/Major+ (n=37), Moderate ( $\mathrm{n}=33$ ), and Minor ( $\mathrm{n}=23)$. Patient demographics and a description of the operations performed are presented in Tables $1 \& 2$ respectively. Statistically significant differences between groups were noted in age (where the age of the patients in the Moderate group were significantly younger than the other groups), American Society of Anesthesiologists grade mix (where all patients undergoing Major/Major+ surgery were assessed as being of grades 2 or 3), operation length (where surgeries of greater severities entailed longer operation lengths) and hospital stay (where surgeries of greater severities entailed longer hospital stays).

\section{Serum cortisol}

When all patients were considered, median baseline ( 8 am) cortisol was 284 [range 53-675] nmol/L, and median peak cortisol was 603 [range 262-1452] nmol/L (Wilcoxon signed rank test, $\mathrm{p}<0.0001$, Figure 1A). One patient undergoing pancreaticojejeunostomy/cholecystojejeunostomy (Major/Major+) was noted to have a baseline 8 am cortisol of $53 \mathrm{nmol} / \mathrm{L}$. This patient mounted a peak cortisol stress response of $435 \mathrm{nmol} / \mathrm{L}$ (821\% response) and underwent the procedure without any complications. Another 15 patients (16\%) had baseline 8 am cortisols between 100-200 nmol/L, and this group exhibited a mean peak cortisol of 457 [range 323-1021] nmol/L.

The peak cortisol positively correlated with the surgical severity: Major/Major+ 680 [range 375-1452], Moderate 581 [range 270-1009], and Minor 574 [range 262-1066] nmol/L (Kruskal-Wallis test, $P=0.0031$, Figure 1B) and the peak cortisol was statistically significantly different between Minor vs Moderate, and Minor vs Major/Major+ (Dunn's multiple comparisons test, $P<0.05$, Figure 1B).

Serum cortisol levels during Minor procedures fluctuated around baseline levels during the procedure. In patients undergoing surgeries classified as Moderate, cortisol levels typically returned to baseline by 8am by 8 hrs after induction. Patients undergoing Major/Major+ surgeries demonstrated total serum cortisol levels comparable to baseline levels on post-operative Days 1-5 (Figures 2A and 2B). 
The median times to developing the peak cortisol level were 2 [range 0-21.5], 4 [range 0-19] and 8 [range 094] hours for Minor, Moderate and Major/Major+ surgeries, respectively (Kruskal-Wallis test, $P=0.0001$ ). With increasing severity, the time taken to achieve the peak cortisol level was longer (Figure 3), and this was significantly different between the Minor vs Major/Major+ and Moderate vs Major/Major+ surgeries (Dunn's multiple comparisons test). However, there was no statistically significant correlation between the duration of surgery and the time to development of the peak cortisol level (Pearson r $0.1857, P=0.0747$ ).

There were weak positive correlations between peak total cortisol and the duration of surgery (Spearman $r$ 0.3202, $\mathrm{p}=0.0018$ ), but not with patient age (Spearman $\mathrm{r} 0.1624, \mathrm{p}=0.1219)$. Patients who developed postoperative complications following Major/Major+ surgery (17/37, 46\%) vs those who did not (20/37, 54\%) had peak postoperative cortisol levels which were not statistically significantly different (respective means \pm S.D. $813 \pm 266 \mathrm{nmol} / \mathrm{L}$ vs $685 \pm 192$, unpaired t-test, $P=0.1092$ ). Although opiates are known to suppress cortisol production ${ }^{13}$, an post-hoc analysis of post-operative cortisol levels on Days 1-5 stratified by opiate use showed that patients receiving opiate analgesia post-operatively (orally, via patient-controlled analgesia, or via epidural, $\mathrm{n}=37$ ) had a significantly higher mean peak post-operative cortisol of $539 \pm 279 \mathrm{nmol} / \mathrm{L}$ compared to those not receiving opiate analgesia $(368 \pm 186 \mathrm{nmol} / \mathrm{L}, \mathrm{n}=42$, unpaired t-test, $\mathrm{P}=0.0017)$. The majority of those who required opiate analgesia were drawn from the Major/Major+ groups, whereas the majority of those who did not require opiate analgesia were drawn from the Minor and Moderate groups. This suggests that the cortisol stress responses on post-operative Days 1-5 were influenced mainly by the severity of the surgery, overcoming any possible inhibition by opiates.

Twenty-three patients were found to have a maximum serum cortisol response of $<480 \mathrm{nmol} / \mathrm{L}$, which is the local threshold for stimulated cortisol levels. The median peak cortisol response in this subgroup was 422 [range 262-475] nmol/L compared to 675 [range 485-1452] nmol/L (unpaired Mann-Whitney test, $P<0.0001$ ) in patients who had an 'adequate' cortisol response $(\geq 480 \mathrm{nmol} / \mathrm{L})$. Baseline $(8 \mathrm{am})$ cortisol was different between the two subgroups: median baseline in $\max <480$ group 231 [range 53-355] vs max $\geq 480$ group 321 [range 111-675], unpaired Mann-Whitney test, $P=0.0001)$. Commensurate with the finding that peak cortisol positively correlated with surgical severity, Major/Major+ surgeries were the predominant type in the $\max \geq 480$ group (Minor 14, Moderate 21, Major/Major+ 35) versus the $\max <480$ group (Minor 9, Moderate 
12, Major/Major+ 2, chi-square test, $P=0.0020)$. Patients who had a peak cortisol response of $<480 \mathrm{nmol} / \mathrm{L}$ did not suffer higher rates of postoperative complications. Three of $23(13 \%)$ patients in the max $<480$ group had post-operative complications versus 17 of $70(24 \%)$ in the $\max \geq 480$ group.

We also performed a subgroup analysis of patients undergoing cholecystectomies, comparing those that underwent laparoscopic procedures ( 9 patients) versus those undergoing open procedures ( 8 patients). The mean \pm S.D. peak cortisol levels were $563 \pm 151 \mathrm{nmol} / \mathrm{L}$ vs $690 \pm 163 \mathrm{nmol} / \mathrm{L}$ respectively, and not significantly different (unpaired t-test, $P=0.1203$ ).

\section{Cortisol binding globulin and free cortisol indices}

Serum cortisol binding globulin $(\mathrm{CBG})$ was measured in 83 patients at baseline (8 am) and 1 hour post induction. Consistent with previous reports ${ }^{4,5} \mathrm{CBG}$ levels dropped acutely during surgery from a mean of $53.6 \pm 10.5 \mathrm{mg} / \mathrm{L}$ at baseline to $43.6 \pm 8.4 \mathrm{mg} / \mathrm{L}$ at 1 hour (paired t-test, $\mathrm{P}<0.0001$ ). There was no significant correlation between the change in cortisol from baseline to 1 hour with the change in CBG over this time (Pearson r $0.1765, P=0.1150)$.

To examine the response in more detail, we measured CBG serially in a subgroup of 18 patients (6 Minor, 6 Moderate, 6 Major/Major+) at baseline, at anaesthetic induction and at 1 hour, 2 hours, 4 hours and 8 hours after induction (Figure 4A). Taking the subgroup as a whole, CBG dropped by $18 \%$ from baseline by 1 hr post induction, with a nadir of $23 \%$ at $4 \mathrm{hr}$ post induction. This drop was similar between the Minor and Moderate groups, but was more marked in the Major/Major+ group at the 4 and $8 \mathrm{hr}$ time points (Figure 4B).

To estimate the free cortisol exposure at each time point, we calculated the free cortisol index (FCI) as the ratio of total cortisol to $\mathrm{CBG}$, a measure that has been shown to correlate well with direct assay of free cortisol

14. Given that there was an acute rise in total cortisol and a drop in CBG in response to surgery, this led to a rise in FCI (Figure 4C) starting from anaesthetic induction from a mean of $5.6 \pm 2.26 \mathrm{nmol} / \mathrm{mg}$ to $12.0 \pm 6.9$ $\mathrm{nmol} / \mathrm{mg}$. We observed a more marked rise in FCI in the Major/Major+ group compared to the Moderate and Minor groups at the $4 \mathrm{hr}$ and $8 \mathrm{hr}$ timepoints post anaesthetic induction (Figure 4D). 


\section{DISCUSSION}

This is the first study to systematically measure and stratify the adrenocortical response to surgery of graded severity; we have investigated a much larger cohort of patients than previously studied for this purpose, and deliberately included patients with surgery of Minor/Moderate level. We found that there was a positive correlation of surgical severity with observed peak serum cortisol, suggesting that the more severe the operation, the greater the provoked stress cortisol response; this effect appears to overcome any inhibitory effect of opiate analgesia post-operatively. Interestingly, we observed that in our cohort of Major/Major+ surgery patients, cortisol levels in post-operative Days 1 to 5 had on average returned to baseline. Other studies on Major/Major+ type surgery (e.g. coronary artery bypass grafting - CABG) have shown more persistent elevations of cortisol at least for post-operative Days 1 and $2^{5}$, and inspection of the data from the four patients that underwent CABG showed that elevated cortisol levels persisted for at least until post-operative Day 4. Although it is possible that insufficient post-operative pain control might explain this persistence of cortisol elevations, a recent study by van Gulik and co-workers suggests that the severity of pain during post-operative care after cardiac surgery does not correlate with changes in cortisol levels ${ }^{15}$. Therefore, the persistence of cortisol elevation may be due to the intrinsic physiological stress of these procedures.

In 1969 Plumpton and Besser examined serum cortisol levels in patients undergoing procedure equivalent to Major/Major+ surgery ${ }^{3}$. The mean peak cortisol response presented herein was $44 \%$ lower compared to the values reported in 1969 (726 vs. $1297 \mathrm{nmol} / \mathrm{L}$ ). The range of peak cortisol responses to Major/Major+ surgery in our study was 375 to $1452 \mathrm{nmol} / \mathrm{L}$, whereas the range reported by Plumpton and Besser was 22 to $75 \mu \mathrm{g} / \mathrm{dl}$ (607 to $2070 \mathrm{nmol} / \mathrm{L}$ ), i.e. $30-38 \%$ lower. Baseline 8 am cortisol was also about 50\% lower in the current study (284 vs. $536 \mathrm{nmol} / \mathrm{L})^{16}$. The Mattingly fluorometric assay for cortisol is non-specific and exhibits a positive bias compared to modern immunoassays, which use highly specific antibodies, and which are aligned to isotope-dilution mass spectrometry reference methods. The only comparative data for the fluorometric assay with immunoassays that exists suggests that there is a mean bias of $+96 \mathrm{nmol} / \mathrm{L}$, i.e. the magnitude of bias is insufficient to explain the difference in cortisol levels observed ${ }^{16}$. However, most of the observed difference is likely to be due to the use of modern anaesthetic and surgical techniques which reduce the amount of physiological stress compared to older techniques. 
Our group, in 2003, has also previously examined the cortisol responses immediately pre-operatively and postoperatively in 31 patients undergoing Major/Major+ surgery in the form of laparotomies. In this study, we found, using a Roche ES700 automated ELISA for cortisol, that the mean pre-operative cortisol level was 453 $\mathrm{nmol} / \mathrm{L}$, rising to $700 \mathrm{nmol} / \mathrm{L}$ post-operatively ${ }^{4}$. The respective ranges for pre-operative cortisol were $88-882$ $\mathrm{nmol} / \mathrm{L}$ and post-operative cortisol $294-1631 \mathrm{nmol} / \mathrm{L}$, i.e. similar cortisol responses to those seen in this study. The 2003 study showed that the CBG fell post-operatively compared to pre-operatively, consistent with our results, leading to more exaggerated increases in free cortisol levels than might be predicted from simple inspection of total cortisol levels. This fall in CBG is due to the secretion of pro-inflammatory cytokines such as IL-6, as well as by the rise in cortisol itself, both of which synergistically suppress CBG expression in hepatocytes ${ }^{17}$. Roth-Isigkeit's study of 2000 showed, in 28 patients undergoing coronary artery bypass surgery (equivalent to Major/Major+), that the mean pre-operative total cortisol was approximately $220 \mathrm{nmol} / \mathrm{L}$, postoperatively the cortisol rose to approximately $1000 \mathrm{nmol} / \mathrm{L}$. In their study, mean CBG fell from approximately $540 \mathrm{nmol} / \mathrm{L}$ to a post-operative nadir of $480 \mathrm{nmol} / \mathrm{L}$ (a drop of $11 \%$ or so), leading to a rise in calculated free cortisol, consistent with our findings ${ }^{5}$.

Our study carries two specific implications. Plumpton and Besser's study also showed that responses of cortisol to surgical stress was replicated by insulin tolerance testing (ITT), which uses the physiological stress of a controlled hypoglycaemia, implying that ITT is suitable to assess the ability of potentially hypoadrenal patients to withstand surgical and other stresses. Their data was used to define an acceptable minimum cut-off for peak cortisol during an ITT at $580 \mathrm{nmol} / \mathrm{L}(21 \mu \mathrm{g} / \mathrm{dl})$, based on their finding that the minimum cortisol response to ITT was $21 \mu \mathrm{g} / \mathrm{dl}$ and the minimum peak cortisol level observed in their cohort of patients undergoing surgery was $22 \mu \mathrm{g} / \mathrm{dl}$. This cut-off has persisted over the last 50 years or so, and most endocrine units now use cut-offs for ITT reduced to take account of the positive bias of the fluorometric assay, for example $500 \mathrm{nmol} / \mathrm{L}^{18}(480$ $\mathrm{nmol} / \mathrm{L}$ in our unit). The same peak cortisol standards are in practice used to interpret other tests commonly used for assessing hypoadrenalism such as the short Synacthen test (SST) ${ }^{19}$. Our data show that that the minimum peak stress cortisol response to Major/Major+ surgery is $375 \mathrm{nmol} / \mathrm{L}$, suggesting that our perception of the minimum acceptable cortisol response will need to be re-defined. In the same vein, El-Farhan and coworkers found that the normative lower reference limit for the peak response to SST using an Abbott Architect 
assay is 416-430 nmol/L, again re-defining standards for the minimum acceptable cortisol response for this test ${ }^{11}$. Our findings suggest that the peak cortisol criteria for evaluating the response to ITT are likely to be lower than the historical cut-offs conventionally used by clinicians, and will need to be revised to take account of the changes in cortisol assay technology over the last 50 years to avoid mis-classifying patients as hypoadrenal, and to avoid inappropriate steroid therapy.

Secondly, the main clinical justification for administering additional oral or parenteral hydrocortisone in hypoadrenal patients undergoing surgery has been to replicate the stress levels of cortisol observed in normal patients undergoing surgery, on the principle that replacement is required to allow such patients to withstand the physiological stress of surgery. The recommendations for the doses to be used vary between authorities, for example European guidelines advocate the administration of $100 \mathrm{mg}$ hydrocortisone intramuscularly before induction of anaesthesia, for surgeries of all severities, whether minor or major. This initial dose is followed by variable courses of enhanced-dose hydrocortisone post-operatively depending on the severity of surgery and as to whether the patient can take oral medication post-operatively ${ }^{20}$. On the other hand, Endocrine Society recommendations suggest that for major surgery, $100 \mathrm{mg}$ hydrocortisone is given intravenously followed by an infusion of $200 \mathrm{mg} / 24 \mathrm{~h}$ or alternatively $50 \mathrm{mg}$ boluses every 6 hours. As for minor to moderate surgery, doses of $25-75 \mathrm{mg} / 24 \mathrm{~h}$ are recommended ${ }^{19}$. In completely hypoadrenal patients, $100 \mathrm{mg}$ intramuscular doses are known to achieve maximal serum concentrations (Cmax) between 1857-4885 nmol/L at Tmax values of between $0.3-3 \mathrm{hr}^{21}$. Pharmacokinetic studies with $20 \mathrm{mg}$ oral doses show a Cmax of $841 \pm 157$ (S.D.) nmol/L at Tmax of $1.2 \pm 0.4 \mathrm{hr}^{22}$. Similarly, Jung and co-workers showed that the Cmax following $20 \mathrm{mg}$ oral doses ranges from 318 to $905 \mathrm{nmol} / \mathrm{L}$ and following $50 \mathrm{mg}$ intravenous doses $1372-$ $4205 \mathrm{nmol} / \mathrm{L}^{23}$. This suggests that doses of $100 \mathrm{mg}$ lead to the development of cortisol levels that vastly exceed the peak levels observed physiologically after even Major/Major+ surgery. This is even more concerning when one considers that the CBG falls during surgery, leading to elevated free cortisol exposure.

Our data supports a stratified approach to dosing hydrocortisone for surgery ${ }^{19,24}$. Taking into consideration our data and the abovementioned pharmacokinetic data, we conclude that lower doses, for example $50 \mathrm{mg}$ intramuscularly or intravenously for Major/Major+ surgery, and $25 \mathrm{mg}$ intramuscularly or orally for Minor/Moderate surgery should be able to cater for stress requirements with a safety margin. Moreover, our 
finding that the physiological levels of cortisol tend to return to baseline by post-operative day 1 even in Major/Major+ surgery would suggest that hydrocortisone doses could be tapered more rapidly than currently suggested by guidelines. In the case of patients undergoing extended periods of recovery and stress postoperatively, such as those undergoing CABG, a low-dose hydrocortisone infusion can be used to maintain cortisol levels for longer periods of time, adjusted to achieve cortisol levels at around the $400-500 \mathrm{nmol} / \mathrm{L}$ range as observed in our cohort ${ }^{20}$. Such a stratified and rapidly tapered approach to supplementation would ideally be tested prospectively to ensure that it was safe and practically feasible.

A limitation of the present study is that it does not include a direct assessment of free cortisol levels, which would ideally be measured using equilibrium dialysis. We did not use equilibrium dialysis as this involves a manual dialysis step, making it impractical in the face of the number of samples analysed in this study. Calculated free cortisol levels based on affinity constants such as Coolens' equation are of limited utility in acutely ill patients ${ }^{25,26}$. We have used pragmatic measures of total cortisol, CBG and FCI, as they are of more practical utility to clinicians. Salivary cortisol may be an alternative measure of free cortisol levels: this has been used in the context of paediatric surgery ${ }^{27}$ and oral surgery ${ }^{28}$, but its utility has not yet been explored in this context. Second, we have not measured ACTH levels, which are known to rise during surgery and general anaesthesia ${ }^{29}$, as this study was intended to assess the cortisol response to inform recommendations for replacement in hypoadrenal patients. Third, we have not made a specific assessment of cortisol metabolism during surgery in this study. One previous study has looked at cortisol metabolism after cardiac surgery using the technique of quantification of urinary cortisol metabolites, and found that surgery was associated with elevated ratios of cortisol metabolites to cortisone metabolites, suggesting that there is a relative reduction in 11ß-hydroxysteroid dehydrogenase (11ß-HSD) activity after Major/Major+ class surgery ${ }^{30}$. Serum cortisol:cortisone is substantially increased after cardiac surgery but this is driven mainly by the increase in cortisol and there appears to be no significant change in cortisone post-operatively, this phenomenon was speculated to be due to an increase in 11ß-HSD1 (cortisone to cortisol) activity vs 11ß-HSD2 (cortisol to cortisone) activity ${ }^{31}$. It is likely that this phenomenon partly underlies the rise in cortisol that we have observed. From the perspective of informing the cortisol replacement of patients with hypoadrenalism, this consideration does not affect the principle of providing hydrocortisone at doses to achieve similar cortisol levels to those observed during elective surgery in euadrenal patients. 
In summary, our study shows that the stress cortisol responses to elective surgery are positively correlated with the operative severity, and that the stress cortisol levels are generally lower than in older studies, but comparable to more recent studies. This suggests that the accepted but historical standards for assessment of hypoadrenal patients during dynamic testing need to be re-evaluated. Pre-operative hydrocortisone doses given to hypoadrenal patients can be reduced from those recommended by some guidelines without compromise to patient safety, to obviate any side effects from overdosage, such as hyperglycaemia, hypernatraemia and superinfection ${ }^{32}$. 


\section{REFERENCES}

1 Desborough, J.P. (2000) The stress response to trauma and surgery. Br J Anaesth 85, 109-117.

2 Gibbison, B., Angelini, G.D. \& Lightman, S.L. (2013) Dynamic output and control of the hypothalamic-pituitary-adrenal axis in critical illness and major surgery. Br J Anaesth 111, 347-360.

3 Plumpton, F.S. \& Besser, G.M. (1969) The adrenocortical response to surgery and insulin-induced hypoglycaemia in corticosteroid-treated and normal subjects. Br J Surg 56, 216-219.

4 le Roux, C.W., Chapman, G.A., Kong, W.M., Dhillo, W.S., Jones, J. \& Alaghband-Zadeh, J. (2003) Free cortisol index is better than serum total cortisol in determining hypothalamic-pituitary-adrenal status in patients undergoing surgery. J Clin Endocrinol Metab 88, 2045-2048.

5 Roth-Isigkeit, A.K., Dibbelt, L. \& Schmucker, P. (2000) Blood levels of corticosteroid-binding globulin, total cortisol and unbound cortisol in patients undergoing coronary artery bypass grafting surgery with cardiopulmonary bypass. Steroids 65, 513-520.

6 Andersson, B., Ansari, D., Norden, M., Nilsson, J. \& Andersson, R. (2013) Surgical stress response after colorectal resection. Int Surg 98, 292-299.

7 Leopold, S.S., Casnellie, M.T., Warme, W.J., Dougherty, P.J., Wingo, S.T. \& Shott, S. (2003) Endogenous cortisol production in response to knee arthroscopy and total knee arthroplasty. J Bone Joint Surg Am 85-A, 2163-2167.

8 Roth-Isigkeit, A.K. \& Schmucker, P. (1997) Postoperative dissociation of blood levels of cortisol and adrenocorticotropin after coronary artery bypass grafting surgery. Steroids 62, 695-699.

9 Taylor, L.K., Auchus, R.J., Baskin, L.S. \& Miller, W.L. (2013) Cortisol response to operative stress with anesthesia in healthy children. J Clin Endocrinol Metab 98, 3687-3693.

10 Le Blanc-Louvry, I., Coquerel, A., Koning, E., Maillot, C. \& Ducrotte, P. (2000) Operative stress response is reduced after laparoscopic compared to open cholecystectomy: the relationship with postoperative pain and ileus. Dig Dis Sci 45, 1703-1713.

11 El-Farhan, N., Pickett, A., Ducroq, D., Bailey, C., Mitchem, K., Morgan, N., Armston, A., Jones, L., Evans, C. \& Rees, D.A. (2013) Method-specific serum cortisol responses to the adrenocorticotrophin test: comparison of gas chromatography-mass spectrometry and five automated immunoassays. Clin Endocrinol (Oxf) 78, 673-680.

12 Copeland, G.P. (2002) The POSSUM system of surgical audit. Arch Surg 137, 15-19.

13 Grossman, A., Gaillard, R.C., McCartney, P., Rees, L.H. \& Besser, G.M. (1982) Opiate modulation of the pituitary-adrenal axis: effects of stress and circadian rhythm. Clin Endocrinol (Oxf) 17, 279-286.

14 le Roux, C.W., Sivakumaran, S., Alaghband-Zadeh, J., Dhillo, W., Kong, W.M. \& Wheeler, M.J. (2002) Free cortisol index as a surrogate marker for serum free cortisol. Ann Clin Biochem 39, 406-408.

15 van Gulik, L., Ahlers, S., van Dijk, M., Bruins, P., Meima, M.E., de Rijke, Y.B., BiemondMoeniralam, H.S., Tibboel, D. \& Knibbe, C.A. (2016) Procedural pain does not raise plasma levels of cortisol or catecholamines in adult intensive care patients after cardiac surgery. Anaesth Intensive Care 44, 52-56.

16 Orme, S.M., Peacey, S.R., Barth, J.H. \& Belchetz, P.E. (1996) Comparison of tests of stress-released cortisol secretion in pituitary disease. Clin Endocrinol (Oxf) 45, 135-140.

17 Emptoz-Bonneton, A., Crave, J.C., LeJeune, H., Brebant, C. \& Pugeat, M. (1997) Corticosteroidbinding globulin synthesis regulation by cytokines and glucocorticoids in human hepatoblastoma-derived (HepG2) cells. J Clin Endocrinol Metab 82, 3758-3762.

18 Erturk, E., Jaffe, C.A. \& Barkan, A.L. (1998) Evaluation of the integrity of the hypothalamic-pituitaryadrenal axis by insulin hypoglycemia test. J Clin Endocrinol Metab 83, 2350-2354.

19 Bornstein, S.R., Allolio, B., Arlt, W., Barthel, A., Don-Wauchope, A., Hammer, G.D., Husebye, E.S., Merke, D.P., Murad, M.H., Stratakis, C.A. \& Torpy, D.J. (2016) Diagnosis and Treatment of Primary Adrenal Insufficiency: An Endocrine Society Clinical Practice Guideline. J Clin Endocrinol Metab 101, 364-389.

20 Husebye, E.S., Allolio, B., Arlt, W., Badenhoop, K., Bensing, S., Betterle, C., Falorni, A., Gan, E.H., Hulting, A.L., Kasperlik-Zaluska, A., Kampe, O., Lovas, K., Meyer, G. \& Pearce, S.H. (2014) Consensus statement on the diagnosis, treatment and follow-up of patients with primary adrenal insufficiency. J Intern Med 275, 104-115.

21 Hahner, S., Burger-Stritt, S. \& Allolio, B. (2013) Subcutaneous hydrocortisone administration for emergency use in adrenal insufficiency. Eur J Endocrinol 169, 147-154.

22 Derendorf, H., Mollmann, H., Barth, J., Mollmann, C., Tunn, S. \& Krieg, M. (1991) Pharmacokinetics and oral bioavailability of hydrocortisone. J Clin Pharmacol 31, 473-476. 
23 Jung, C., Greco, S., Nguyen, H.H., Ho, J.T., Lewis, J.G., Torpy, D.J. \& Inder, W.J. (2014) Plasma, salivary and urinary cortisol levels following physiological and stress doses of hydrocortisone in normal volunteers. BMC Endocr Disord 14, 91.

24 Kehlet, H. (1975) A rational approach to dosage and preparation of parenteral glucocorticoid substitution therapy during surgical procedures. A short review. Acta Anaesthesiol Scand 19, 260-264.

25 Coolens, J.L., Van Baelen, H. \& Heyns, W. (1987) Clinical use of unbound plasma cortisol as calculated from total cortisol and corticosteroid-binding globulin. J Steroid Biochem 26, 197-202.

26 Molenaar, N., Groeneveld, A.B. \& de Jong, M.F. (2015) Three calculations of free cortisol versus measured values in the critically ill. Clin Biochem 48, 1053-1058.

27 Hsu, A.A., von Elten, K., Chan, D., Flynn, T., Walker, K., Barnhill, J., Naun, C., Pedersen, A.M., Ponaman, M., Fredericks, G.J., Crudo, D.F. \& Pinsker, J.E. (2012) Characterization of the cortisol stress response to sedation and anesthesia in children. J Clin Endocrinol Metab 97, E1830-1835.

28 Miller, C.S., Dembo, J.B., Falace, D.A. \& Kaplan, A.L. (1995) Salivary cortisol response to dental treatment of varying stress. Oral Surg Oral Med Oral Pathol Oral Radiol Endod 79, 436-441.

29 Newsome, H.H. \& Rose, J.C. (1971) The response of human adrenocorticotrophic hormone and growth hormone to surgical stress. J Clin Endocrinol Metab 33, 481-487.

30 Vogeser, M., Felbinger, T.W., Roll, W. \& Jacob, K. (1999) Cortisol metabolism in the postoperative period after cardiac surgery. Exp Clin Endocrinol Diabetes 107, 539-546.

31 Vogeser, M., Groetzner, J., Kupper, C. \& Briegel, J. (2003) The serum cortisol:cortisone ratio in the postoperative acute-phase response. Horm Res 59, 293-296.

32 Sprung, C.L., Annane, D., Keh, D., Moreno, R., Singer, M., Freivogel, K., Weiss, Y.G., Benbenishty, J., Kalenka, A., Forst, H., Laterre, P.F., Reinhart, K., Cuthbertson, B.H., Payen, D., Briegel, J. \& Group, C.S. (2008) Hydrocortisone therapy for patients with septic shock. N Engl J Med 358, 111-124. 
Table 1. Patient demographics.

\begin{tabular}{|c|c|c|c|c|c|}
\hline & $\begin{array}{c}\text { All } \\
(n=93)\end{array}$ & $\begin{array}{c}\text { Major/Major+ } \\
(\mathbf{n}=\mathbf{3 7})\end{array}$ & $\begin{array}{c}\text { Moderate } \\
(\mathbf{n}=\mathbf{3 3})\end{array}$ & $\begin{array}{l}\text { Minor } \\
(\mathbf{n}=23)\end{array}$ & Statistical comments \\
\hline Age (yrs) & $56.1[51.0-60.6]$ & $62.5[54.1-66.3]$ & $41.2[34.1-51.0]$ & $60.6[48.5-68.0]$ & $\begin{array}{c}P<0.0001 \text { (Kruskal- } \\
\text { Wallis) } \\
*, o\end{array}$ \\
\hline Male:Female & $46: 47$ & $22: 15$ & $17: 16$ & $7: 16$ & NS (chi-square) \\
\hline BMI $\left(\mathrm{kg} / \mathrm{m}^{2}\right)$ & $27.4[26.0-28.7]$ & 26.4 [24.4-28.9] & $27.5[26.0-32.0]$ & $27.8[23.0-30.9]$ & NS (Kruskal-Wallis) \\
\hline SBP $(\mathbf{m m H g})$ & $128 \pm 21$ & $132 \pm 19$ & $123 \pm 15$ & $129 \pm 28$ & NS (one-way ANOVA) \\
\hline Heart rate $($ bpm) & $75 \pm 12$ & $74 \pm 12$ & $77 \pm 12$ & $73 \pm 14$ & NS (one-way ANOVA) \\
\hline $\operatorname{ASA}(1: 2: 3)$ & 9:67:17 & $0: 26: 11$ & $7: 24: 2$ & $2: 17: 4$ & $P=0.009$ (chi-square) \\
\hline Median op length (min) & $150[120-180]$ & $270[240-345]$ & $125[100-150]$ & $75[60-90]$ & $\begin{array}{c}P<0.0001 \text { (Kruskal- } \\
\text { Wallis) } \\
*, \dagger, \circ \\
\end{array}$ \\
\hline Median Hosp. Stay (days) & $2[2-3]$ & $7[7-10]$ & $1[1-2]$ & $1[1-1]$ & $\begin{array}{c}P<0.0001 \text { (Kruskal- } \\
\text { Wallis) } \\
\uparrow^{\circ}\end{array}$ \\
\hline Laparoscopic & $19(21 \%)$ & $3(8 \%)$ & $13(39 \%)$ & $3(14 \%)$ & \\
\hline $\begin{array}{l}\text { Post-op analgesia } \\
\text { (None/non-opioids: Oral opiates: Epidural: } \\
\text { PCA: Ketamine) }\end{array}$ & $49: 12: 21: 20: 3$ & $5: 3: 20: 19: 3$ & $25: 6: 1: 1: 0$ & 19:3:0:0:0 & \\
\hline \multicolumn{6}{|l|}{ Specialty } \\
\hline UGI & $10(11 \%)$ & $7(18 \%)$ & $2(6 \%)$ & $1(4 \%)$ & \\
\hline HPB & $34(37 \%)$ & $23(64 \%)$ & $10(30 \%)$ & $1(4 \%)$ & \\
\hline Gen & $6(6 \%)$ & 0 & $4(12 \%)$ & $2(9 \%)$ & \\
\hline H\&N & $37(40 \%)$ & $1(3 \%)$ & $17(52 \%)$ & $19(83 \%)$ & \\
\hline CT & $6(6 \%)$ & $6(15 \%)$ & 0 & 0 & \\
\hline
\end{tabular}

Median [95\% confidence interval for median] shown for Age, body mass index (BMI), operation length, hospital stay. Mean \pm S.D. shown for systolic blood pressure (SBP) and heart rate. Where shown in the right hand column: NS = no significant difference; * = significant difference between Minor and Moderate group (Dunn's multiple comparison test, alpha $=0.05) ; \dagger=$ significant difference between Minor and Major/Major+ group (Dunn's multiple comparison test, alpha $=$ $0.05) ;{ }^{\circ}=$ significant difference between Moderate and Major/Major+ group (Dunn's multiple comparison test, alpha $=0.05$ ). Note that some patients in the Major/Major+ group had multimodal post-op analgesia in some cases (for example epidural plus PCA).

ASA, American Society of Anesthesiologists grade; PCA, patient-controlled analgesia; UGI, upper gastrointestinal; HPB, Hepato-Pancreato-Biliary; Gen, General; $\mathrm{H} \& \mathrm{~N}$, Head and Neck; CT, Cardiothoracic 
Table 2. Description of operations. *Note that two patients had a combined liver ablation/resection and open cholecystectomy and have been counted twice.

\begin{tabular}{|c|c|}
\hline Major/Major+ $(\mathrm{n}=37)$ & \\
\hline Open liver resection or ablation* & 8 \\
\hline Open cholecystectomy* & 8 \\
\hline Open coronary artery bypass & 4 \\
\hline Open Whipple's procedure & 4 \\
\hline Open transthoracic oesophagectomy & 3 \\
\hline Open gastrectomy & 2 \\
\hline Open pancreatectomy \& splenectomy & 2 \\
\hline Distal gastrectomy, duodenal resection, Roux-en-Y bypass & 1 \\
\hline Open coronary artery bypass, aortic $\&$ mitral valve replacement & 1 \\
\hline Open mitral valve replacement & 1 \\
\hline Open neck dissection and mediastinal clearance & 1 \\
\hline Open pancreaticojejunostomy & 1 \\
\hline Laparoscopic pancreaticojejunostomy \& cholecystojejunostomy & 1 \\
\hline Laparoscopic gastric bypass & 1 \\
\hline Laparoscopic Whipple's procedure & 1 \\
\hline \multicolumn{2}{|l|}{ Moderate $(\mathrm{n}=33)$} \\
\hline Open thyroidectomy/thyroid lobectomy & 17 \\
\hline Laparoscopic cholecystectomy & 9 \\
\hline Open abdominal hernia repair & 2 \\
\hline Laparoscopic appendectomy & 2 \\
\hline Open de-roofing of liver cyst & 1 \\
\hline Laparoscopic Heller's cardiomyotomy & 1 \\
\hline Laparoscopic sleeve gastrectomy & 1 \\
\hline \multicolumn{2}{|l|}{ Minor $(\mathbf{n}=\mathbf{2 3})$} \\
\hline Parathyroidectomy & 19 \\
\hline Diagnostic laparoscopy & 3 \\
\hline Liver ablation & 1 \\
\hline
\end{tabular}




\section{FIGURES}

Figure 1. (A) Baseline (filled circles) and peak (filled triangles) cortisol levels. Individual values plotted. Middle line is median. $* * * * P<0.0001$, Wilcoxon matched-pairs signed rank test. (B) Peak cortisol response according to surgical severity (Minor: filled circles; Moderate: filled squares; Major/Major+: filled triangles). Individual values plotted. Middle line indicates median. $\mathrm{P}=0.0031$ for difference (Kruskal-Wallis test), * $P<0.05$, Dunn's multiple comparison test.

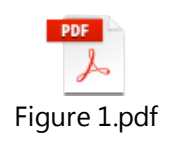

Figure 2. Perioperative cortisol response for patients classified by surgical severity (Minor: filled circles, dotted lines; Moderate: filled squares, dashed lines; Major/Major+: filled triangles, solid lines). (A) Serum total cortisol values (medians with error bars representing interquartile range). (B) Values normalised to 8am baseline cortisol on the day of operation (medians plotted with error bars representing interquartile range).

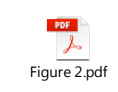

Figure 3. Time to developing the peak cortisol level (hours) according to surgical severity (Minor: filled circles; Moderate: filled squares; Major/Major+: filled triangles). Individual values plotted. Line indicates the median time. $P<0.0001$ for difference, $*$ and $* * *$ denote significant differences using Dunn's multiple comparisons test.

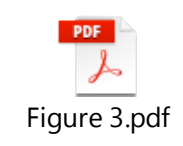

Figure 4. Perioperative cortisol binding globulin (CBG) and free cortisol (FCI) response for subgroup of 18 patients with consecutive CBG measurements. (A) mean $( \pm$ S.D.) absolute CBG values $(\mathrm{mg} / \mathrm{L})$ plotted. (B) normalised (\%) to baseline 8 am value, and separated into different surgical severities (Minor: filled circles, dotted lines; Moderate: filled squares, dashed lines; Major/Major+: filled triangles, solid lines). Comparison, using Tukey's multiple comparisons test, of Minor vs Major/Major + : $\uparrow P<0.05 ; \dagger \uparrow P<0.01 ; \dagger+P<0.001$; $\uparrow \dagger$ $P<0.0001$; comparison of Moderate vs Major/Major+: * $P<0.05$; ** $P<0.01$; *** $P<0.001$; **** $P<0.0001$. (C) absolute FCI values (nmol/mg) for subgroup of 18 patients with consecutive CBG measurements, * $P<0.05$ 
for comparison to $\mathrm{T}=0$ (Holm-Sidak multiple comparison test). (D) FCI response separated into surgical severities (Minor: filled circles, dotted lines; Moderate: filled squares, dashed lines; Major/Major+: filled triangles, solid lines). Comparison of Minor vs Major/Major+ $\dagger \dagger P<0.0001$ (Tukey’s multiple comparisons test).

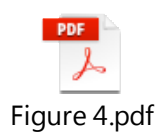

